\title{
Co-design methods for eliciting patient needs for wrist splint design
}

Charlotte Pyatt ${ }^{\mathrm{a}}$, Matt Sinclair ${ }^{\mathrm{a}} *$ and Richard Bibb ${ }^{\mathrm{a}}$

${ }^{a}$ School of Design \& Creative Arts, Loughborough University, Loughborough, United Kingdom

*Corresponding author. Email: m.sinclair@lboro.ac.uk

M. Sinclair ORCiD: 0000-0002-6427-134X

R. Bibb ORCiD: 0000-0002-3975-389X

6993 words including Abstract, tables, references and captions. 


\section{Biographical Notes}

Dr. Charlotte Pyatt is a Design Researcher at DCA Design International. Her academic research investigates the role of product design in patient compliance, specifically focussing on personalisation and co-design. She brings expertise in co-design to her current role at DCA, incorporating co-creation and participatory methods into professional practice.

Dr. Matt Sinclair is Programme Director for Industrial Design at the School of Design \& Creative Arts, Loughborough University. His research investigates the future of manufacturing, and in particular how digital technologies enable the increasing involvement of the consumer in the design and manufacture of their own products.

Richard Bibb is Professor of Medical Applications of Design and Associate Dean Research at the School of Design and Creative Arts, Loughborough University. His research explores the application of advanced design techniques in medical devices, prosthetics, orthotics and surgery resulting in more than 100 peer reviewed articles. 


\title{
Co-design methods for eliciting patient needs for wrist splint design
}

\author{
Wrist splints are a common treatment for rheumatoid arthritis, however their \\ effectiveness is compromised by patients not wearing splints as prescribed. Previous \\ research has identified reasons for non-compliance, but typically lacks insights that \\ could lead to improved splint design. A three-part study using design probes, context \\ mapping and a personalization toolkit as co-design methods for eliciting patient needs \\ for wrist splint designs, is described. It identifies three themes and nine sub-themes for \\ situations that affect compliance in wearing a splint. Additionally four motivating \\ factors to wear, and 10 motivating factors not to wear a splint are presented. Nine \\ requirements for improved splint design are established and form the basis of the design \\ for a prototype personalization toolkit. Testing of this toolkit reveals participants are \\ keen to wear splints whose appearance matches the clothes they are wearing or \\ activities being undertaken. Co-design methods are shown to be capable of identifying \\ determinants of compliance not previously discussed in the literature, as well as \\ eliciting patient-specific needs for splint design.
}

Keywords: probes, personalization, toolkits, co-design, rheumatoid arthritis

\section{Introduction}

Wrist splints are well-established in the treatment of Rheumatoid Arthritis (RA), but it is widely reported that their effectiveness is compromised by poor patient compliance (Spoorenberg, Boers and van der Linden 1994a; Callinan and Mathiowetz 1996; Taylor, Hanna and Belcher 2003; Sandford, Barlow and Lewis, 2008; Veehof et al. 2008b). This paper reports the use of a co-design research methodology to investigate poor compliance.

Specifically, it aims to demonstrate how co-design methods have been successful at

(1) identifying the relevant determinants behind why participants do or do not wear wrist splints,

(2) communicating participants' desires for a future wrist splint design. 
RA is a chronic inflammatory disease characterized by joint swelling, joint tenderness and destruction of synovial joints, leading to disability (Aletaha et al. 2010), and is the most common type of autoimmune arthritis (Rudeman and Tambar 2011). It is 3 times more common in women than men (Symmons et al. 2002), with 1 to $3 \%$ of women suffering from RA in their lifetime (Rudeman and Tambar 2011). Borenstein, Silver and Jenkins (1993) describe treatment as a 'multi-layered pyramid' where education, occupational therapy, rest and nonsteroidal anti-inflammatory drugs (NSAIDs) represent the base. Treatment often involves splints that 'apply external forces to an extremity in order to manipulate specific anatomical structures for a desired effect,' (Taylor, Hanna and Belcher 2003) by supporting and immobilizing affected joints. However, poor compliance observed in clinical practice has been attributed to patient dissatisfaction due to splints:

- Causing pain

- Being unwieldy

- Being aesthetically unappealing

- Being difficult to put on/take off

- Getting wet/dirty

- Not fitting correctly

While the literature describes poor compliance, it has focused on quantitative measures of frequency and duration of wear and paid less attention to how splints are experienced by users or how this knowledge might lead to the design of improved splints. In this research, qualitative co-design methods such as design probes, interviews and context mapping were employed to gain a deeper and more nuanced understanding of participants' relationships with their splints. The work is based on the premise that digital design and manufacturing processes can produce bespoke, personalized splints on demand (Paterson 
2013; Kelly, Paterson and Bibb 2016). A prototype customization toolkit was developed, based on Paterson's (2013) design (Figure 1), which utilizes multi-material 3D printing to create soft areas and compensate for localized swelling. However, rather than providing tools for the clinician, this research investigated the specification and use of a mass customization toolkit (Tseng and Jiao 1998) to incorporate patient choice into the design of splints. Finally, the paper discusses the self-reported expectations regarding compliance of participants who used the toolkit.

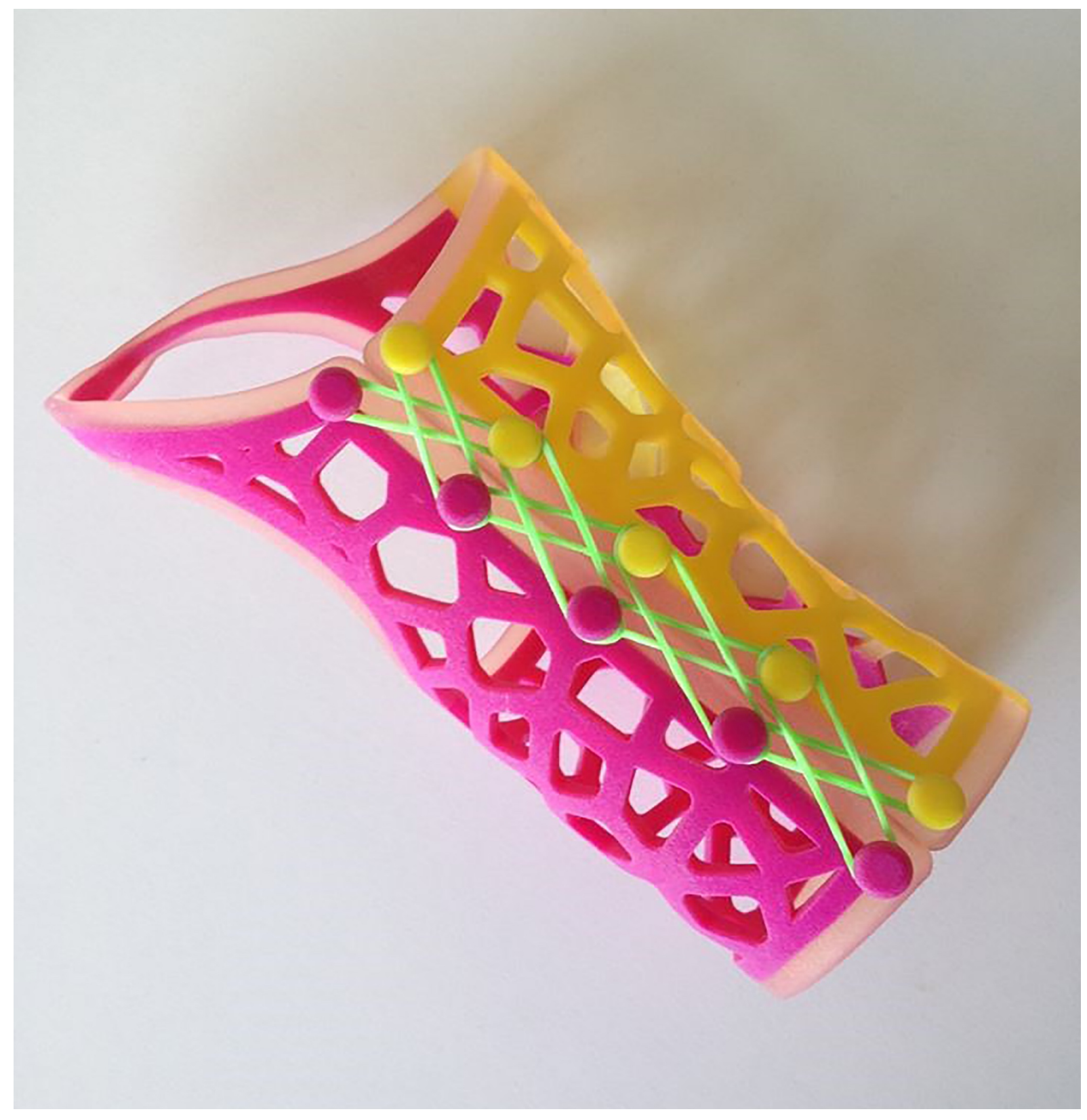

Figure 1. Multi-material wrist splint by Paterson 


\section{Approach in clinical literature}

Previous investigations of wrist splint compliance have typically taken a quantitative and statistical approach, generating findings lacking in rich, insightful data that consider patient needs capable of informing the design process. However, a small number of studies have attempted to take a patient-centred approach through the use of questionnaires, interviews and diaries.

\section{Questionnaires}

Agnew \& Maas (1995) used fixed response questionnaires in a survey of 265 RA patients to determine compliance and its influencing variables, showing that discomfort was a prominent contributor. However, a follow-up survey of 130 patients given a new, more comfortable splint showed no increase in compliance. Agnew and Maas were unable to account for this, possibly due to questions that focused only on activities (driving, gardening, typing, etc.) and ignored context or experience. This corresponds to criticisms of fixed response questionnaires that argue that 'the researcher prepares not only the questions, but also the answers he or she expects to get, leaving 'little possibility for initiative on the part of the respondent' (Sanders and Stappers 2012). Similarly Spoorenberg, Boers and van der Linden (1994b) used openended and multiple-choice questionnaires with 44 RA patients to assess wrist splint use. However, the usefulness of the results was limited as questions were based on the researchers' prior knowledge.

\section{Structured and semi-structured interviews}

Feinberg and Brandt (1981) conducted interviews with 50 RA patients to identify the frequency of wearing and reasons for cessation of use. Follow up interviews were then carried out 3 to 34 months after prescription. However, this study relied on participants' ability to recall information over an extended period and failed to account for discrepancies in accuracy 
of recall. As with Agnew and Maas (1995), little attempt was made to understand participants' relationships with their splint. Oakes et al. (1970) conducted interviews with 66 patients to investigate the effect of family expectations on compliance. However, participants were reviewed at 6-month intervals, meaning again that accuracy was reliant on recall over an extended period. Unlike previous work, Veehof et al's (2008a) study of 18 RA patients aimed to generate qualitative data relating to participants' experiences, knowledge and opinions. Conducting semi-structured interviews in participants' own homes allowed participants to behave and respond less formally than if interviewed in a clinic. Nonetheless, this study was able only to access an 'explicit' level of knowledge and can be criticized on the grounds that 'what people say is different to what they do' (Sanders and Stappers 2012). While it investigated how users behaved, it was less successful at revealing the reasons why this behaviour occurred.

\section{Self-report diaries}

Criticisms of self-report diaries are pertinent as expectations of family and clinician are known influencers of compliance (Oakes et al. 1970; Veehof et al., 2008b). The dangers of misreporting to please the researcher, or changing behaviour in order to show the diarist in a good light (Robson 2011) must be considered. Callinan and Mathiowetz (1996) used selfreport diaries to compare hard and soft resting hand splints among 39 RA patients but did not account for misreporting and considered data generated from the diaries in much less detail than that from other methods. Similarly, Veehof et al. (2008b) investigation of the efficacy of working splints with 33 RA patients, which used self-report diaries to measure how long participants wore splints each day, failed to consider the tendency to misreport. In both cases, the diary structure prevented issues being explored deeply. Improvements to this method might have been the use of reflective journals to 'ask participants to provide an account of their experiences in a particular situation and reflect on that experience' (Robson 2011), or 
photo/video diaries that would have allowed researchers to observe participants wearing splints, though neither would have fully resolved the possibility of misreporting.

\section{Co-design approach}

Previous qualitative methods have failed to yield insightful data that considers the role of patient motivation and social expectation on compliance. Importantly, previous studies lacked recommendations capable of informing the design process to generate products that improve compliance. In this paper, a co-design approach was used for the first time, allowing participants 'to feel that they are the experts in the experience domain being explored' (Visser et al. 2005).

Participatory approaches are recognized as changing the way that functional and emotional user needs are understood by designers (Sánchez de la Guía et al. 2017). In healthcare, co-design is acknowledged as enabling patients to 'share their specialist forms of expertise' (Robert et al. 2015, Donetto et al. 2015) and incorporate these insights into new proposals. Of particular relevance to this study, Rijn and Stappers (2007) used interview, observation and generative techniques to co-design a language-learning toy for autistic children. They then used context mapping to extract 'experiences, feelings and dreams' from parents, who were given a sensitization workbook to complete at home, in order to 'access deeper levels of knowledge [when reflecting] on their experiences in a moment that really suits them'. Glasemann and Kanstrup (2011) used similar methods to explore how mobile technology could be developed with young diabetics, telling participants that 'they were the designers of future diabetes technology'. A toolkit/creativity pack with materials to facilitate design activity were given to participants and user mock-ups were 'chosen to facilitate the young people's emotional expressions'. Debrah et al., (2017) described the use of design probes and toolkits to identify the healthcare needs of mothers in South Africa and Ghana, arguing that participatory methods have an emancipatory outcome in promoting healthier 
living through the sharing of patients' expertise. Similarly Hussain and Sanders (2012) used generative tools to co-design prosthetic legs with Cambodian children, using paper dolls 'to encourage children to talk about aesthetic preferences and self-image'. Finally, Wilson et al. (2015) explored co-design techniques for giving people with aphasia a voice in the development of therapy tools, recommending 'creating tangible design languages... using images, gestures, demonstrations and physical artefacts'.

\section{Co-design methods used for understanding compliance}

This study was conducted in three stages, utilizing different co-design methods in each:

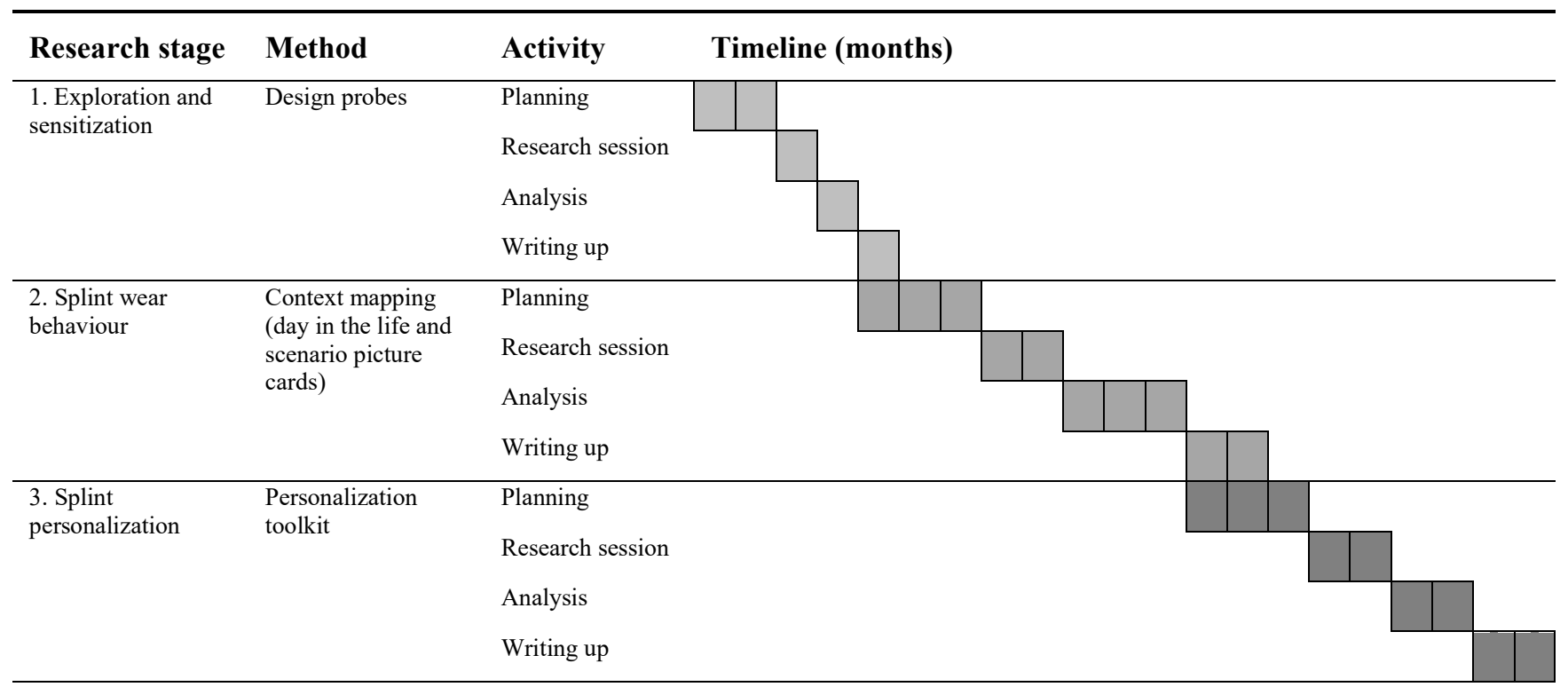

Table 1. Stages of research and co-design tools applied

A purposive, convenience sampling strategy was used for this research. The same sample of participants was used for all three stages (Table 1), therefore extra effort was required to keep participants engaged; this was achieved by making sessions enjoyable, emphasizing the importance of participants' contribution, and maintaining regular communication between studies. In addition, the empathic approach taken (see Discussion) contributed to low dropout. Two participants withdrew before Stage 2 (one moved to a distant location and one gave no reason); two additional participants withdrew before Stage 3, (one due to illness and one gave 
no reason). Whilst using the same participants in all stages introduced the risk of the sample size decreasing, the saturation of results shows that this had limited impact. Participant profiles are given in Table 2 below.

\begin{tabular}{|c|c|c|c|c|c|c|c|c|}
\hline $\begin{array}{l}\text { Participant } \\
\text { code }\end{array}$ & $\begin{array}{l}\text { Years } \\
\text { since } \\
\text { diagnosis }\end{array}$ & $\begin{array}{l}\text { Years } \\
\text { wearing } \\
\text { splints }\end{array}$ & $\begin{array}{l}\text { Splint type } \\
\text { (resting or } \\
\text { working) }\end{array}$ & Age & Gender & Study 1 & Study 2 & Study 3 \\
\hline S1001 & 3.5 & 0.5 & Working & 62 & Female & Yes & Yes & Yes \\
\hline S1002 & 15 & 15 & Working & 53 & Female & Yes & Yes & \\
\hline S1006 & 2 & 2 & Both & 59 & Female & Yes & & \\
\hline S1007 & 15 & 15 & Working & 59 & Female & Yes & Yes & Yes \\
\hline S1010 & 2 & 2 & Both & 38 & Female & Yes & Yes & Yes \\
\hline S1012 & 12 & 9 & Both & 41 & Female & Yes & Yes & Yes \\
\hline S1013 & 6 & 5 & Both & 49 & Female & Yes & & \\
\hline S1014 & 26 & 26 & Both & 28 & Female & Yes & Yes & Yes \\
\hline S1017 & 6 & 4 & Both & 49 & Female & Yes & Yes & \\
\hline
\end{tabular}

Table 2. Participant demographics

\section{Research Ethics}

All research was conducted in accordance with Loughborough University's Ethical Advisory Committee guidance on research with human participants. This first required the completion and approval of an ethics checklist. A participant information sheet was sent to participants before each stage, who were advised they could withdraw from the study, without reason, at any point. As research was conducted in participants' homes, a female chaperone also attended. Consent to publish anonymized data was received from all participants with data retained in accordance with Loughborough University's (2019) Data Protection Policy. The 
ethics checklist and participant information sheets are available at https://doi.org/ 10.17028/rd.lboro.9715340.

\section{Research Stage 1: Exploration and sensitization}

Stage 1 aimed to sensitize participants to the features and objectives of the study and provide inspiration. Design Probes are design-oriented user research toolkits based on selfdocumentation (Mattelmäki 2005). Their value lies in 'facilitating communication and gaining insights into what users might perceive to be relevant in their lives' (Debrah et al. 2017). Probes were used for three reasons: to 'shake the preconceptions' (Gaver et al. 2004) about the target group and what was already known from the literature; to build a relationship and trust by 'creating a dialogue between participants and themselves' (Herd, Bardill and Karamanoglu 2009); and to sensitize participants by inviting them to 'reflect on and verbalize their experiences, feelings and attitudes and to visualize their actions and contexts' (Mattelmäki 2005).

There are no rules for applying design probes (Herd, Bardill, and Karamanoglu 2009). However, they should 'control the direction, in which the user should unconsciously go' (Lundgaard and Larsen 2007), in order to prepare them for subsequent research. In this project, probes contained five tasks to be completed over five days, taking participants through a journey of discovery about themselves and sensitizing them for future research (Figure 2). The contents were:

(1) About you - questions and photos

(2) Arthritis and splints - questions and photos

(3) Day in the life - photo diary

(4) Personalization - questions and photos

(5) Future splint - design activity 

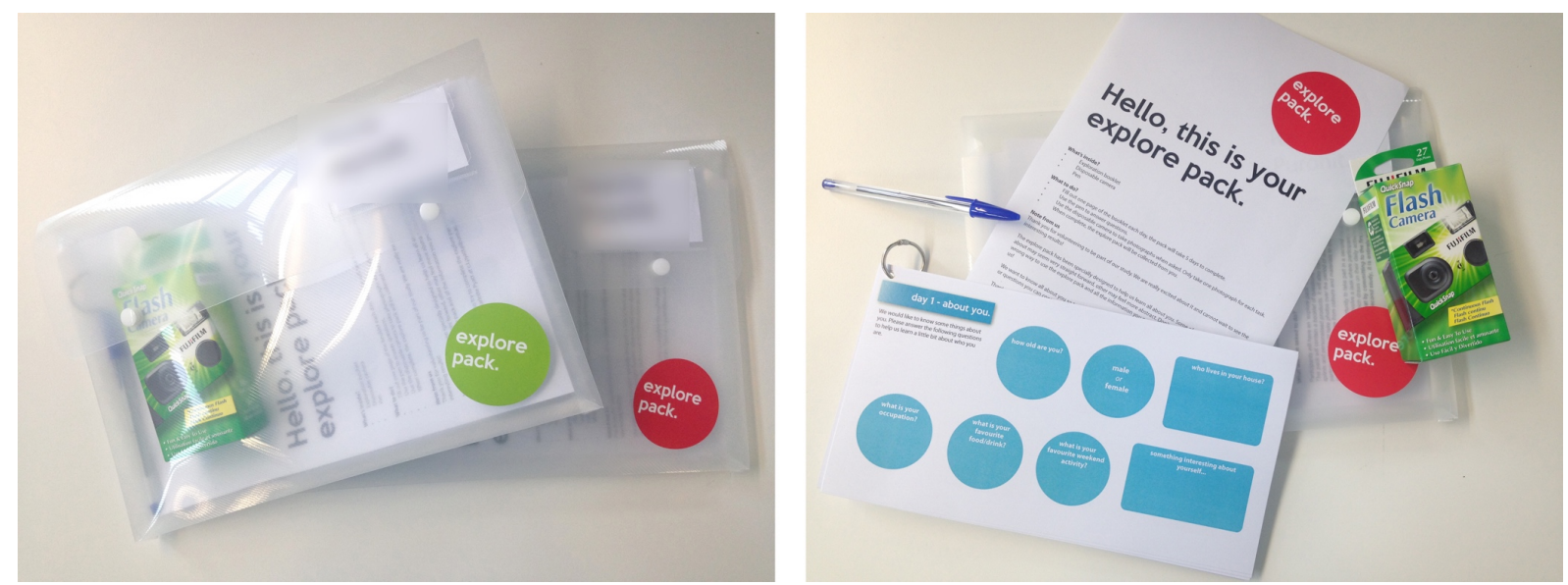

Figure 2. (a) The design probes assembled. (b) The design probe contents.

The probes were delivered to the participants' homes so that the researcher could meet them and begin a personal relationship to be maintained over the 18 months duration of the project. Meeting participants in person allowed the researcher to explain the probes, answer questions and overcome issues that arise when directly involving users in unfamiliar design activities (Groeneveld et al. 2019). In particular these meetings established trust in the integrity of the researcher and the approach, which Warwick (2017) argues is especially important. On completion of the five-day task, the researcher collected the probes.

Mattelmäki (2005) suggests that completed probes are discussed with participants to gain insights. However, in this study the purpose of the probes was primarily to inform stages 2 and 3, which were intended to reveal insights in a more targeted way. In addition, the probes were intended to help establish a relationship with participants in preparation for the following studies, and to sensitize participants to the subject. For this reason, the probes were analyzed without further input from participants.

Mattelmäki (2006) refers to making sense, outlining or interpretation as more appropriate terms than analysis, discussing the process of extracting meaning through emerging themes, patterns or clusters of affinitive information. Questions were posed to guide 
the interpretation such as 'what do we know about participants experience of wearing splints?' and 'what do we know about participants desires for a future wrist splint?' Following approaches outlined by Braun and Clarke (2006), data was initially coded in relation to each of the questions. Since the data set was relatively small, coding was performed manually using Post Its. Once coding was complete, this approach allowed the Post Its to be arranged into themes, which revealed themselves rather than being driven by the literature or researchers' preconceptions.

Two key findings resulted from the probes. Firstly, data showed situations where participants would or would not wear their splints. Looking beyond these places and activities, themes were identified (Table 3) that gave reasons for participants' behaviour; for example, removing the splint when cleaning.

\begin{tabular}{ll}
\hline Themes & Sub-themes \\
\hline Everyday tasks & Wet/messy tasks \\
& Achievable tasks \\
& Restrictive tasks \\
Socializing & Does want to be seen \\
& Does not want to be seen \\
Resting & In bed \\
& Do not need splint \\
& Rest from wearing splint \\
\hline
\end{tabular}

Table 3. Splint wear behaviour themes taken from stage 1

The probes also gave insight into what participants' ideal splint might be like, with the most common desires relating to appearance, hygiene and comfort:

- More colours and patterns 
- Match clothes

- More comfortable

- Washable/not dirty easily

- Waterproof

- More than one

- Breathable

- More support

- Velcro alternative

\section{Research Stage 2: Splint wear behaviour}

Stage 2 investigated participants' motivations for wearing and not wearing splints, along with their aspirations for future splints. Traditional design research techniques such as observation and self-report diaries were judged to be inappropriate at this stage because of issues relating to participant privacy and lack of control for comparison across the group.

Context mapping, used for understanding how people interact with products (Visser et al. 2005), was selected. Participants were required to map the wearing of their current splint over two different days using a timeline adapted from the 'day in the life' exercise outlined in Convivial Toolbox: Generative Research for the Front End of Design (Sanders and Stappers 2012). Participants completed the timeline based on a typical weekday and a typical weekend day, and discussed their behaviour on these days (Figure 3). The 'path of expression' (Sanders and Stappers 2012) encouraged participants to consider 'present experiences, good and bad memories from the past and hopes and dreams for the future' (Sanders and Stappers 2014). This was achieved through four phases:

- Everything they did that day from waking to going to sleep

- Whether they wore their splint or not at each point in the day (tick or cross) 
- How they felt at each point in the day (happy or sad face)

- Reasons for their actions and what aspect of splints made them behave the way they $\operatorname{did}$
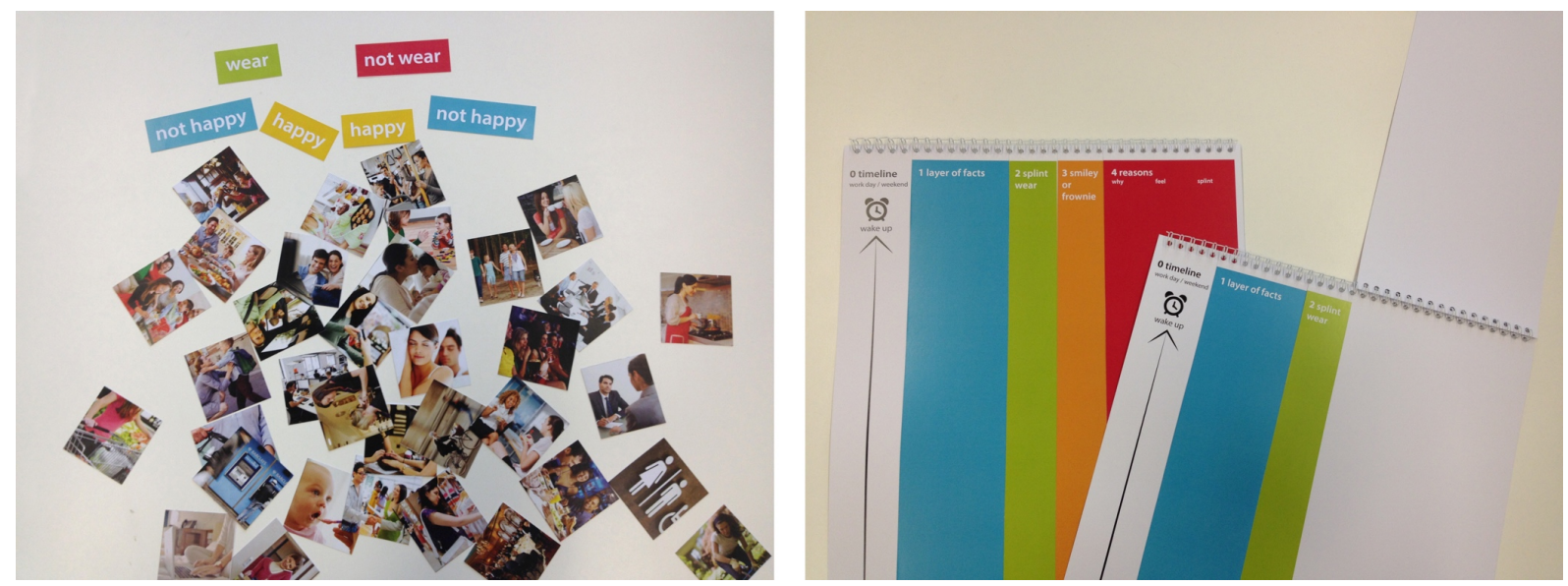

Figure 3. (a) Day in the life timeline

(b) Scenario picture cards

To explore the effect of social situations, a scenario picture card task elicited information that may not arise by studying only two particular days. Scenario picture cards are an application of card sorting used in participatory and user centred design (Cockbill 2017), defined as a 'telling' activity in which participants use cards to organize, categorize and prioritize ideas (Sanders, Brandt and Binder 2010). Participants grouped cards displaying activities from grocery shopping to a formal dinner, based on whether they would 'wear' or 'not wear' their splint and whether they would be 'happy' or 'not happy' (Figure 4a, 4b). Participants were asked to select cards from each group and tell stories about their experiences. These stories were gathered verbally, recorded and transcribed. 

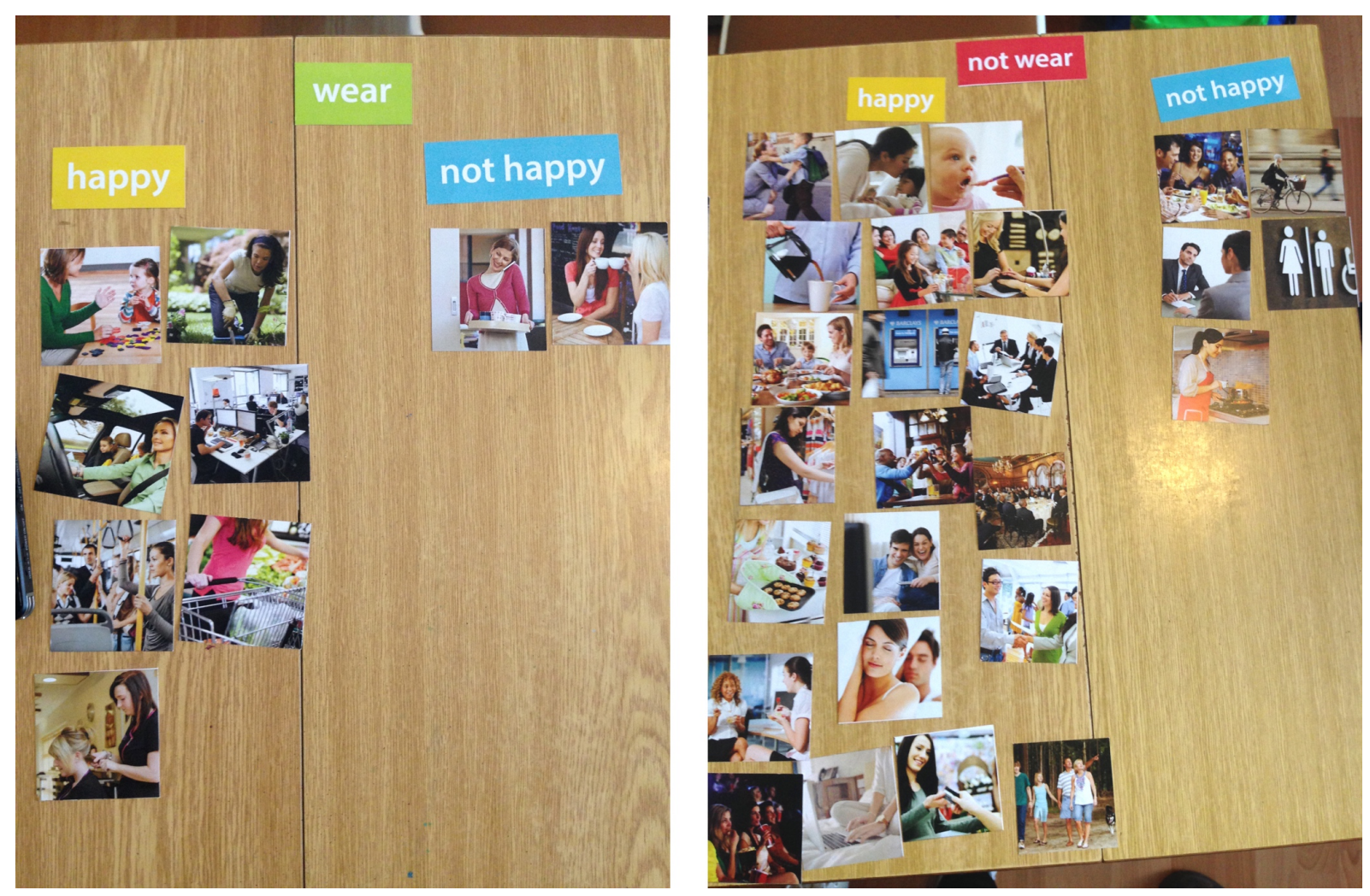

Figure $4 \mathrm{a}, 4 \mathrm{~b}$. Scenario picture cards put into groups by a participant

Data was analyzed using thematic analysis guided by the research questions identified in the Introduction. Nvivo (ver.12) was used for analysis and coding as this was a larger data set than in stage 1. Data was coded into predefined groups, however, the themes within these groups were coded inductively (Bradley, Curry \& Devers, 2007) using a bottom up approach. This ensured that codes revealed themselves and were not guided by the literature or preconceptions. Coding was performed until no new concepts emerged and text for each code was compared to ensure they reflected the same concept. Table 4 shows the themes identified when studying why participants wear or do not wear splints. An interactive PDF of the connections between themes (motivators) is available at https://doi.org/10.17028/rd.lboro.9715517. 


\begin{tabular}{ll}
\hline Motivators to wear splints & Motivators to not wear splints \\
\hline Practical/help with tasks & Practical issues with the splint \\
Condition pain/swelling & Do not need to wear \\
Keep doing things in the future & Negative social reactions \\
No reason to remove & Break from wearing \\
& Alternative way to do things \\
& Does not help achieve task \\
& Appearance \\
& Negative feelings \\
& Do not have splint on them \\
& Do not want to wear \\
\hline
\end{tabular}

Table 4. Motivators to wear or not wear wrist splints taken from stage 2

After being asked to provide reasons for their behaviour, participants were better able to express their needs and wishes for a future splint design; summarized as

- Discreet/blend in

- Less restricting/more flexibility

- Bigger colour range

- More attractive

- Match outfit

- Wipe clean/washable

- Better sizes/bespoke fit

- Less bulky

- Less medical in appearance 


\section{Research Stage 3: Splint personalization}

The final stage explored the effects of splint appearance using a prototype customization toolkit. Toolkits are a well-established means of enabling user engagement in the customization of products, typically requiring the user to choose pre-determined options to create a product that matches their requirements (Tseng and Jiao 1998). When engaged in customization activities, users can be regarded as performing co-design activities (Piller et al. 2005). However, customization toolkits are typically complex software programs that are difficult to simulate when there is a requirement to show customized changes realistically, in real-time. To overcome this limitation, the rendering software package KeyShot was used to produce a prototype toolkit (Figure 5). This gave the ability to provide participants with a real-time visualization of their customized splint. Prior to testing, a variety of wrist splint CAD models were created following the technique established by Paterson (2013). 3D pointcloud scan data of a typical user's hand and wrist were used as the basis for an initial CAD model; this model was split into regions (to allow different colours and materials to be assigned) and a number of cut out patterns were mapped to the surfaces of the model. The CAD models were then imported into KeyShot and parameters set up to enable participants to change the pattern, colour and surface finish from a variety of predefined options.

During testing, participants were first asked to co-design a single wrist splint (Figure 6). This was to see how participants would prioritize needs where a clinician can only offer a single splint. Participants were then asked to create as many splint designs as they desired. This was to see how the opportunity of having multiple splints affected the way participants codesigned them. After the exercise, semi-structured interviews were conducted to understand the choices made. 


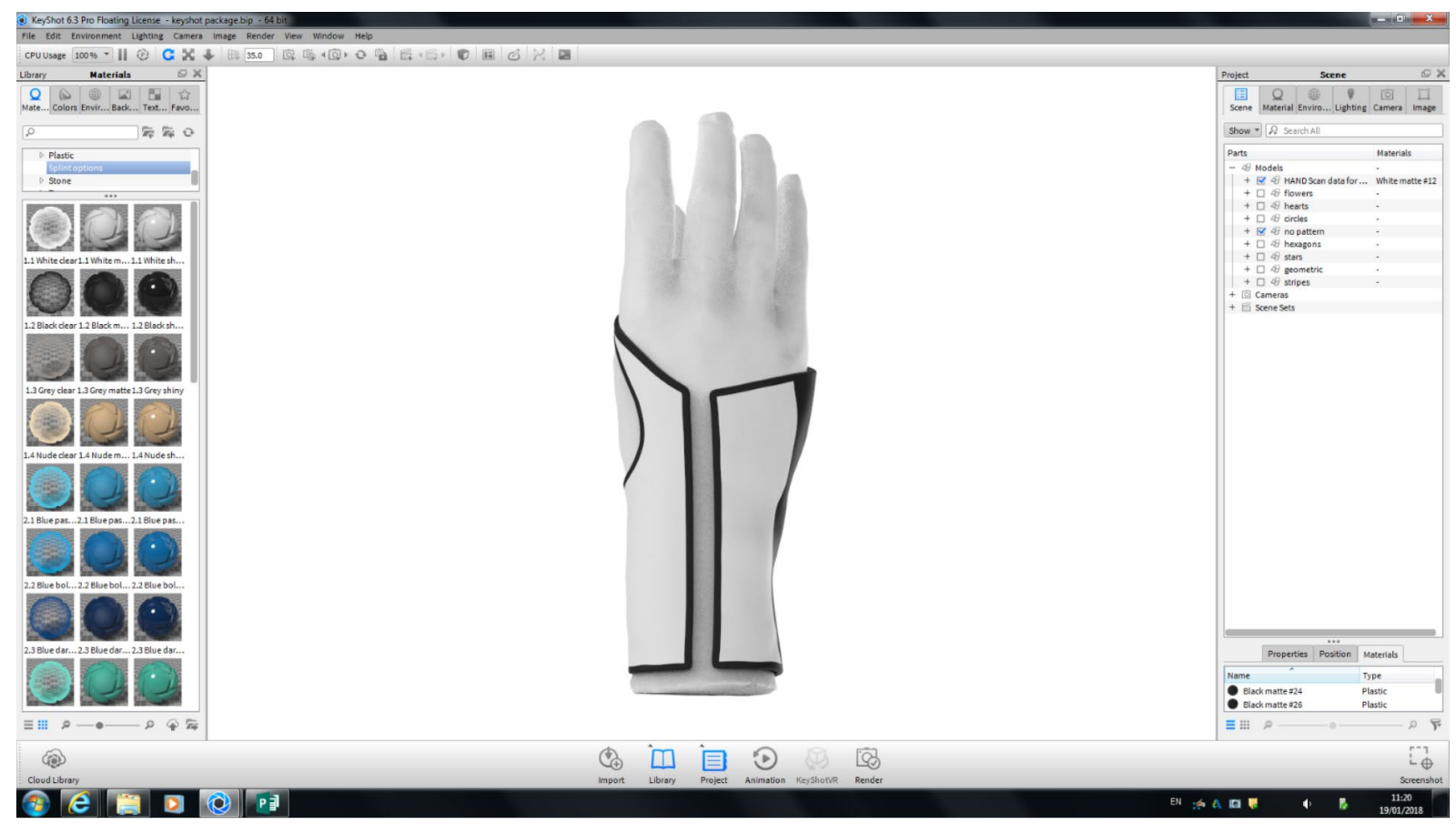

Figure 5. The personalization toolkit

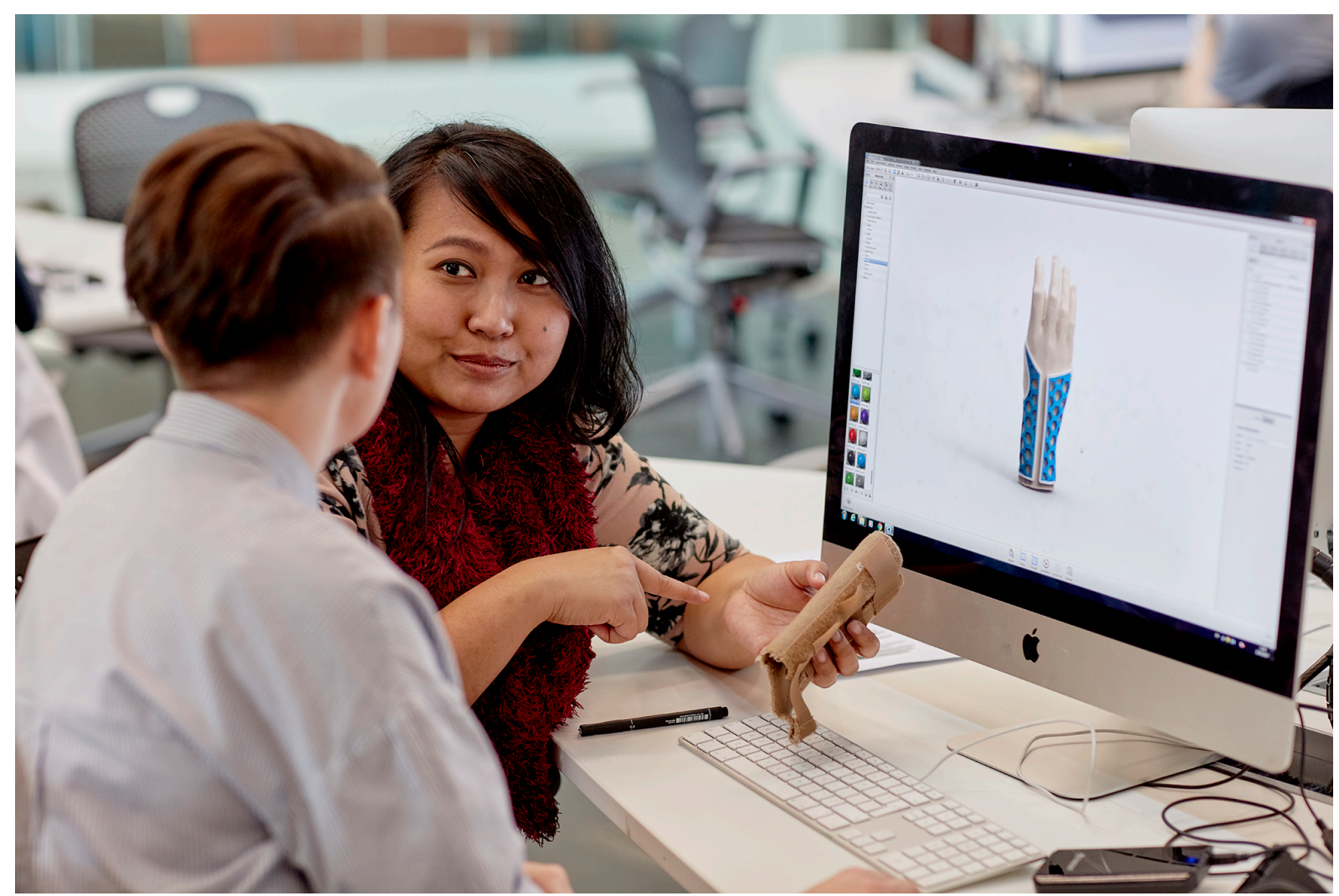

Figure 6. An example user testing the personalization toolkit

Finally, the scenario picture cards were reintroduced to allow a comparison of splint wear between participants' current splints and the ones they had co-designed. The picture cards 
were set out for each participant in the groups they had made in stage 2. Participants were then asked to regroup the picture cards based on the personalized splints they had created. Where changes were observed, participants were questioned about the reasons for their changes. In situations where participants indicated they would be willing to wear their personalized splint but would not have worn their current splint, this was attributed to being more comfortable with the appearance of the personalized splint, and it being designed more specifically for the situation on the card. There were no situations in which participants stated they would wear their current splint but not the personalized splint. In situations where participants indicated they would not wear their current or personalized splint, this was mainly attributed to functional issues where improved appearance would have no influence on the scenario.

The key finding in Stage 3 was that participant needs are best met by multiple splint designs for specific scenarios, rather than a 'one design fits all' approach.

\section{Impact of co-design}

\section{Understanding splint wear behaviour}

Through co-design methods, this research has led to a deeper understanding of participants' motivations to wear and not wear their wrist splints. As suggested in the literature, it was found that practical issues such as the splint getting wet, dirty and being restrictive was the most prevalent motivator for participants to not wear splints. However, unlike previous studies, it also revealed that social interactions have a significant effect on participants' splint wear, with all participants stating they would remove their splint to avoid negative reactions. Moreover, whilst the effect of splint appearance on patient compliance is mentioned in the literature (Spoorenberg, Boers and van der Linden et al., 1994; Agnew and Maas, 1995; Van Lede, 2002; Taylor, Hanna and Belcher et al., 2003; Veehof et al., 2008b), there has 
previously been little evidence to support it. In this research all participants said that a splint's appearance was reason enough to not wear it in certain situations, with reasons including a 'scruffy' appearance (dirty, soiled, stained, worn), not matching a chosen outfit and not looking attractive.

Co-design methods provided an understanding of participants' complete experience of wearing a splint, focusing not only on negative aspects but also discovering why participants are motivated to wear splints. Participants were more likely to wear a splint if they needed support to complete a task, if they were in pain, and if it enabled them to continue activities they enjoyed. These motivators to wear have been overlooked in the literature, yet provide insights into what drives patients to wear splints, which could be useful in generating compliance strategies. Participants were also able to more easily evoke their emotions. Sanders and Stappers (2012) state that 'our emotional states have a large influence, not only on how we feel but also on how we think and act.' However, participants' feelings regarding the wearing of wrist splints have been neglected in previous work. By using generative research methods, participants were better equipped to discuss how they felt about wearing splints, and were able to express feelings of frustration, embarrassment and difference to their peers when wearing splints. Conversely, participants expressed much more positive feelings towards the splints they had co-designed in Stage 3 of the study, relating feelings such as increased confidence, openness to positive social reactions and the power to overcome stigma.

The methods also enabled participants to predict their behaviour for a future splint design. The 'path of expression' technique used to steer participants through 'the successive considering of present experiences, good and bad memories from the past, and hopes and dreams for the future' (Sanders and Stappers 2014) enabled participants to reflect on their current and past behaviour. This better equipped participants to suggest their needs for a future splint grounded in their own experiences. For example, in stage 2, many participants 
recalled instances where they have either not worn or been unhappy wearing their splint due to receiving negative comments. In response participants commented that a future splint should be discreet, and were able to make suggestions such as a splint that matched their clothes, a splint that could roll away up their arm, to be quickly hidden, or one that better matched their skin tone. Stage 3 saw all participants co-designing splints using colours that would match their clothes and as a result they predicted they would be happier wearing these splints in the future.

\section{Inspiring a future splint design}

Unlike previous work, this research empowered participants to discuss what they want from a future splint design. One of the main findings from the co-design activities was the idea of multiple splints. When given unlimited choice, it was found that participants typically chose to have between three and four splints, which they could change depending on a given situation. For example, 'everyday', 'night out' (Figure 7) and 'work' splints were common choices amongst participants. Other splints were co-designed for situations specific to that participant, for instance two participants designed splints for when they spend time with their children.

While splint personalization has been suggested previously, this has been from the medical 'expert's' perspective, for example putting the patient's name on the splint or choosing the colour. Co-design revealed that such personalization is unlikely to have a positive effect on compliance, and that participants view splints in a similar way to clothes. Commonalities observed across participants' splint designs show that while 'everyday' splints look different to one another, they are often inspired by participants' favourite colours or patterns that they felt matched their personality. 'Night-out' splints generally had metallic colours and shiny finishes (like jewellery). In contrast, 'work' splints had a smart, professional presentation, 
with dark colours, matte finishes and subtle patterns. These commonalities suggest that future splints, co-designed with patients and fit for specific situations, might lead to increased compliance.
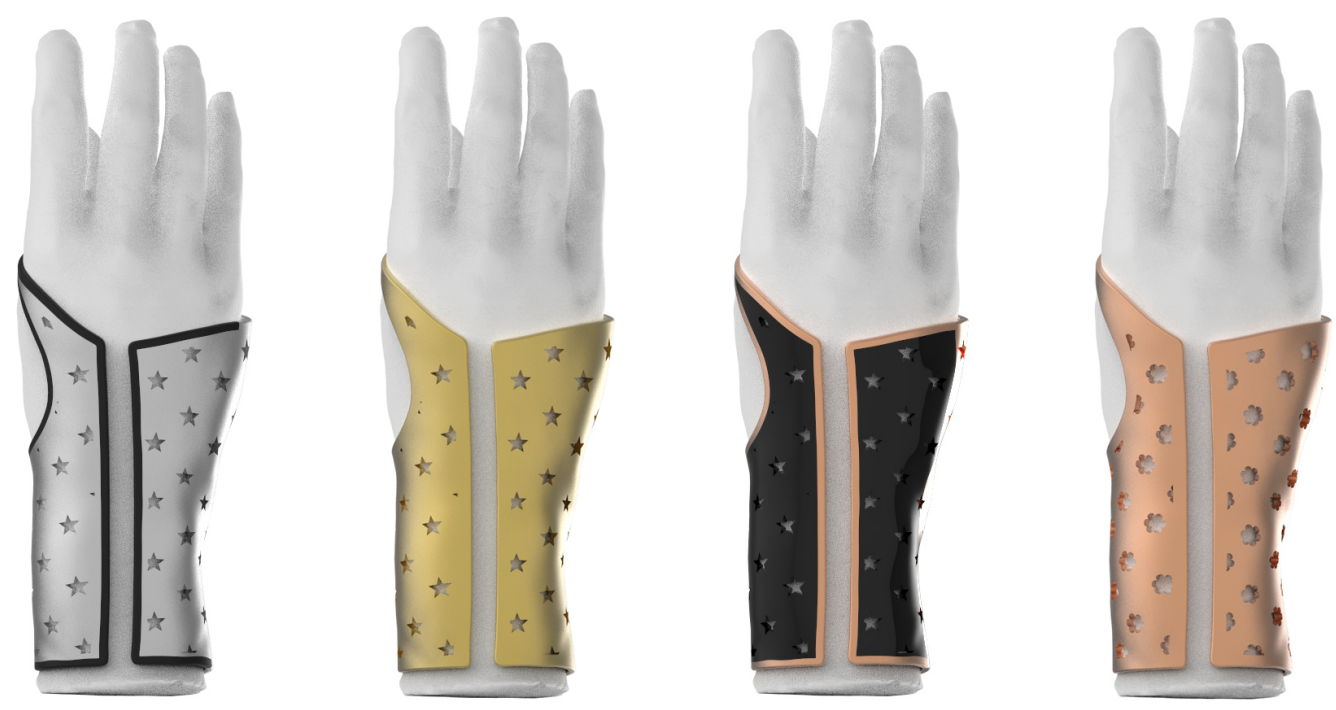

Figure 7. Night out splints co-designed by 4 participants

\section{Discussion: Reflections on the methods}

Quantitative methods used in previous research have not produced actionable insights and have failed to accurately report participants' lived experiences. While the co-design methods employed in this study require a greater investment in time, their value lies in the ability to reveal individual participants' personal experience that is absent from previous work.

Design probes were valuable in establishing dialogue and trust between participants and researcher. It was integral to the research that participants understood that the researcher was not there to judge their actions, but to listen and understand their experiences. This empathic approach (Niinimäki and Koskinen 2011) allowed the researcher to more fully appreciate users' lived experiences, values and relationships with their splints. The benefits were apparent in stage 2 , where participants were willing to honestly report their behaviour and offer stories that allowed the researcher to empathize with their situation. 
The requirement for participants to recall and give reasons for behaviour that they would usually engage in without conscious consideration was a challenge. The timeline activity, particularly implementing the path of expression, was successful at guiding participants through their thought process so they could understand their own actions. Whilst this activity was repetitive, it was successful at conditioning participants to the task, making it easier to express actions, feelings and reasons.

A further challenge was gaining a wide perspective of how participants behaved in different situations during one, relatively short, session. Here the use of scenario picture cards provided an opportunity for participants to comment and organize their thoughts across different scenarios. The success of this method was primarily due to participants having completed the timeline activity first, which trained them to quickly recall and give reasons for their behaviour. However, a limitation of the picture card method is that scenarios are predefined, with the risk that some scenarios may not be relevant to a participant, or a scenario which is important to a participant may not be presented.

The value of the picture cards was emphasized when assessing participants' predicted behaviour change. During stage 2, participants used cards to create groups of images depicting when they would and would not wear their current splints; this was done without being influenced by future splint ideas or the personalization toolkit. After completing the personalization activities in stage 3, participants were able to regroup the cards to reflect their predicted splint wear for the personalized splint(s). This enabled a comparison between stages 2 and 3, offering insights into ways that personalized splints might influence compliance. It is important to emphasize however, that participants grouped cards based on self-predicted splint wear, and that actual future behaviour might differ.

All participants showed excitement towards the personalization toolkit, and all were able to use it competently after guidance. An unexpected outcome was the distraction of 
material choice. Participants were asked to not consider material properties (as these would influence the physical properties of the splint, which was not being studied). However, participants still questioned the material and made assumptions such as 'if the splint has a shiny finish I'm assuming it is a waterproof material'. In addition, two participants requested a colour or pattern that was not available in the toolkit, which, while not affecting the results of the study, gave further insight into what participants desired from their splint designs.

Of note throughout the research was participants' ability to engage in creative activity and come up with ideas. The stage 1 'ideal splint' task asked participants to choose from colour, pattern and material options. As well as this, participants were given a free space to draw their own ideas. None of the participants used drawing but some wrote their ideas, indicating that participants were put off by tasks such as drawing that fell outside their 'comfort zone', indicating the importance of providing opportunities for participants to express themselves in other ways (what Sanders (2006) refers to as 'scaffolds for creativity'). In stage 2, participants considered what a future splint would be like if they were to want to wear it. Because participants had previously been required to identify when splints were not worn, and the reasons for this behaviour, they were able to list features they would like to see in a new splint design. Finally, participants demonstrated creativity in the personalization task. An inspiration sheet was provided, however, almost all participants neglected it and none copied any designs from the inspiration sheet.

\section{Conclusions and Further Research}

As NHS England has stated, there is a "need to shift towards a more personalized approach to health and care so that people have the same choice and control over their mental and physical health that they have come to expect in every other part of their life. And as local health and care organizations work together more closely than ever before, they are recognizing the power of individuals as the best integrators of their own care" (NHS England 2019. p.2). This 
research aligns with such an ambition, demonstrating how co-design methods can be successfully applied to develop a richer understanding of patient compliance and elicit participant desires.

Co-design and generative design research methods enabled participants to uncover how they feel about splints and reasons for wearing or not wearing. The methods allowed a much deeper approach and uncovered determinants not previously discussed in the literature. For instance an important, though somewhat unexpected, insight was participants' desire to reframe the perception of wrist splints, seeing them not as medical interventions but as adornments or clothing. In this reframed context, colours, materials and patterns become key drivers of the design of the splint, rather than superficial additions intended to 'prettify' the device. Additionally, by de-emphasizing the medical nature of the device, participants are arguably distancing themselves from the role of care receiver, instead seeing themselves as individuals who choose to wear items 'tailored' to their physical attributes and abilities.

The co-design activities gave participants tools to express their desires for a future splint providing insight into the number of splints participants want for different situations. Commonalities seen across the splint designs can guide the design of future splints. The customization toolkit suggested that benefits recognized in mass customization literature apply to healthcare. Such toolkits allow users to engage in the creative exploration of preferred choices, in a low cost and low risk manner, unconstrained by time and in an environment (their own home) in which they are likely to feel more comfortable (compared to a doctor's clinic or surgery). However, further research is required to understand patients' willingness to engage with splint customization toolkits when acting alone without guidance. Further research is also recommended to investigate the value of personalization in wrist splints, and whether this value comes from participants engaging in the personalization process itself, the output of the personalization process, or both. 


\section{Acknowledgments}

We would like to thank Loughborough School of Design \& Creative Arts for funding this research and Abby Paterson for her kind contribution of scanned 3D hand data.

\section{Disclosure Statement}

No potential conflict of interest is reported by the authors.

\section{References}

Agnew, Patricia J., and Frederick Maas. 1995. "Compliance in Wearing Wrist Working Splints in Rheumatoid Arthritis." The Occupational Therapy Journal of Research / American Occupational Therapy Foundation 15 (3): 165-80.

Aletaha, Daniel, Tuhina Neogi, Alan J. Silman, Julia Funovits, David T. Felson, Clifton O. Bingham, Neal S. Birnbaum, et al. 2010. "2010 Rheumatoid Arthritis Classification Criteria: An American College of Rheumatology/European League Against Rheumatism Collaborative Initiative." Arthritis and Rheumatism 62 (9): 2569-81.

Borenstein, David G., Gary Silver, and Ellen Jenkins. 1993. “Approach to Initial Medical Treatment of Rheumatoid Arthritis." Archives of Family Medicine 2 (5): 545-51.

Bradley, E., L. Curry, and K. Devers. (2007). Qualitative Data Analysis for Health Services Research: Developing Taxonomy, Themes, and Theory. Health Services Research, 42(4), pp.1758-1772.Treatment of Rheumatoid Arthritis.” Archives of Family Medicine 2 (5): 545-51.

Braun, V. and V. Clarke. 2006. Using thematic analysis in psychology. Qualitative research in psychology. Jan 1; 3(2): 77-101.

Callinan, Nancy J, and Virgil Mathiowetz. 1996. "Soft Versus Hard Resting Hand Splints in Rheumatoid Arthritis: Pain Relief, Preference, and Compliance.” The American Journal of Occupational Therapy 50: 347-53.

Cockbill, Stuart. 2017. "The Role of Co-Design in Supporting Energy-Related Retro Fit by Householders." Available from: https://dspace.lboro.ac.uk/2134/24888

Debrah, R.D., R. De la Harpe and M.K. M'Rithaa. 2017. Design probes and toolkits for healthcare: Identifying information needs in African communities through service design. The Design Journal, 20 (1): S2120-S2134. 
Donetto, S., P. Pierri, V. Tsianakas, and G. Robert. 2015. Experience-based co-design and healthcare improvement: realizing participatory design in the public sector. The Design Journal, 18 (2): 227-248.

Feinberg, Judy, and Kenneth D Brandt. 1981. "Use of Resting Splints by Patients with Rheumatoid Arthritis." American Journal of Occupational Therapy 35: 173-78.

Gaver, William, Andy Boucher, Sarah Pennington, and Brendan Walker. 2004. "Cultural Probes and the Value of Uncertainty." Interactions - Funology.

Glasemann, Marie, and Anne Marie Kanstrup. 2011. "Emotions on Diabetes: A Design Case of User Mock-Ups by Young People Living with Diabetes.” CoDesign 7 (2): 123-30.

Groeneveld, Bob, Tessa Dekkers, Boudewijn Boon \& Patrizia D’Olivo (2018). “Challenges for design researchers in healthcare." Design for Health 2(2): 305-326.

Herd, Kate, A. Bardill, and M. Karamanoglu. 2009. "X-Ray Specs, Stickers and Colouring in: Seeing beyond the Configurator Using Design Probes." Proceedings of the 5th International Conference on Mass Customization and Personalization. 4-8 November: Helsinki, Finland.

Hussain, Sofia, and Elizabeth B.-N. Sanders. 2012. "Fusion of Horizons: Co-Designing with Cambodian Children Who Have Prosthetic Legs, Using Generative Design Tools.” CoDesign 8 (1): 43-79.

Kelly, Sarah, Abby Paterson, and Richard Bibb. 2016. "A review of wrist splint designs for additive manufacture". In Proceedings of 2015 14th Rapid Design, Prototyping and Manufacture conference (RDPM 14), Loughborough, Great Britain, 15-16 Dec. 2015.

Lede, Paul van. 2002. "Minimalistic Splint Design: A Rationale Told in a Personal Style." Journal of Hand Therapy 15 (2): 192-201.

Loughborough University. 2019. “Loughborough University Data Protection Policy” [online]. Available from: https://www.lboro.ac.uk/admin/ar/policy/dpact/ludpp/index.htm Lundgaard, Leena, and Ulrick Martin Larsen. 2007. “Applying Probes As an Inspirational Research Tool for Fashion Design," Nordes 2.

Mattelmäki, Tuuli. 2005. “Applying Probes-from Inspirational Notes to Collaborative Insights." CoDesign 1 (2): 83-102.

Mattelmäki, Tuuli. 2006. “Design Probes.” Helsinki: University of Art And Design NHS England. 2019. Universal Personalised Care: Implementing the Comprehensive Model. Available from: https://www.england.nhs.uk/publication/universal-personalised-careimplementing-the-comprehensive-model/ 
Niinimäki, K. and I. Koskinen. 2011. "I love this dress, it makes me feel beautiful! Empathic knowledge in sustainable design." The Design Journal, 14 (2): 165-186.

Oakes, Thomas W, John R. Ward, Robert M. Gray, Melville R. Klauber, and Philip M. Moody. 1970. "Family Expectations and Arthritis Patient Compliance to a Hand Resting Splint Regimen.” Journal of Chronic Diseases 22 (11): 757-64.

Paterson, Abby. 2013. "Digitalisation of the Splinting Process: Exploration and Evaluation of a Computer Aided Design Approach to Support Additive Manufacture.” Doctoral Thesis, available from: https://dspace.lboro.ac.uk/dspace-jspui/handle/2134/13021

Piller, F; P. Schubert. M. Koch, and Möslein, K. 2005. "Overcoming Mass Confusion: Collaborative Customer Co-Design in Online Communities.” Journal of ComputerMediated Communication 10 (4).

Rijn, H van, and Pieter Jan Stappers. 2007. “Codesigning LINKX: A Case of Gaining Insight in a 'difficult-to-Reach User Group."' IASDR'07 International Association of Societies of Design Research, 1-14.

Robert, Glenn, Jocelyn Cornwell, Louise Locock, Arnie Purushotham, Gordon Sturmey, and Melanie Gager. 2015. "Patients and staff as codesigners of healthcare services" BMJ 350: g7714.

Robson, Colin. 2011. Real World Research. Third Edit. West Sussex: John Wiley \& Sons Ltd.

Rudeman, Eric, and Siddharth Tambar. 2011. "Rheumatoid Arthritis (RA)." American College of Rheumatology, 1-6.

Sánchez de la Guía, L., Puyuelo Cazorla, M. and de-Miguel-Molina, B., 2017. Terms and meanings of "participation" in product design: From "user involvement" to "codesign”. The Design Journal, 20 (1): S4539-S4551

Sanders, Elizabeth B -N. 2006. "Scaffolds for building everyday creativity". In Design for effective communications: Creating Contexts for Clarity and Meaning. Frascara, J. (ed). New York: Allworth Press.

Sanders, Elizabeth B -N., Eva Brandt, and Thomas Binder. 2010. "A framework for organizing the tools and techniques of participatory design." In Proceedings of the 11th biennial participatory design conference, 195-198. ACM.

Sanders, Elizabeth B -N., and Pieter Jan Stappers. 2012. Convivial Toolbox: Generative Research For The Front End Of Design. Amsterdam: BIS.

Sanders, Elizabeth B -N., and Pieter Jan Stappers. 2014. "Probes, Toolkits and Prototypes: Three Approaches to Making in Codesigning." CoDesign 10 (1): 5-14. 
Sandford, Fiona, Nicola Barlow, and Jeremy Lewis. 2008. “A Study to Examine Patient Adherence to Wearing 24-Hour Forearm Thermoplastic Splints after Tendon Repairs." Journal of Hand Therapy 21 (1): 44-52.

Spoorenberg, A., M. Boers, and S. Van der Linden. 1994a. "Wrist Splints in Rheumatoid Arthritis: What Do We Know about Efficacy and Compliance?" Arthritis Care and Research 7 (2): 55-57.

Spoorenberg, A, M. Boers, and S. Van der Linden. 1994b. "Wrist Splints in Rheumatoid Arthritis: A Question of Belief?" Clinical Rheumatology 13 (4): 559-63.

Symmons, D., G. Turner, R. Webb, P. Asten, E. Barrett, M. Lunt, D. Scott, and A. Silman. 2002. "The Prevalence of Rheumatoid Arthritis in the United Kingdom: New Estimates for a New Century.” Rheumatology 41 (7): 793-800.

Taylor, E., J. Hanna, and H.J.C.R. Belcher. 2003. "Splinting of the Hand and Wrist." Current Orthopaedics 17 (6): 465-74.

Tseng, Mitchell M., and Jianxin Roger Jiao. 1998. "Concurrent Design for Mass Customization.” Business Process Management Journal 4 (1): 10-24.

Veehof, Martine, Erik Taal, Marjanne J. Willems, and Mart A.F.J. van de Laar. 2008a. "Determinants of the Use of Wrist Working Splints in Rheumatoid Arthritis." Arthritis and Rheumatism 59 (4): 531-36.

Veehof, Martine, Erik Taal, Heijnsdijk-Rouwenhorst, and Mart A.F.J. van de Laar. 2008b. "Efficacy of wrist working splints in patients with rheumatoid arthritis: a randomized controlled study." Arthritis Care \& Research 59 (12) 1698-1704.

Visser, Froukje Sleeswijk, Pieter Jan Stappers, Remko van der Lugt, and Elizabeth B -N. Sanders. 2005. “Contextmapping: Experiences from Practice.” CoDesign 1 (2): 119 49.

Warwick, L., 2017. Designing Trust: the importance of relationships in social contexts. The Design Journal, 20 (1): S3096-S3105.

Wilson, Stephanie, Abi Roper, Jane Marshall, Julia Galliers, Niamh Devane, Tracey Booth, and Celia Woolf. 2015. “Codesign for People with Aphasia through Tangible Design Languages." CoDesign 11 (1): 21-34. 
